\title{
Sustainable Design of a Nearly Zero Energy Building Facilitated by a Smart Microgrid
}

\author{
Gandhi Habash, ${ }^{1}$ Daniel Chapotchkine, ${ }^{1}$ Peter Fisher, ${ }^{2}$ Alec Rancourt, ${ }^{2}$ \\ Riadh Habash, ${ }^{2}$ and Will Norris ${ }^{3}$ \\ ${ }^{1}$ Azrieli School of Architecture and Urbanism, Carleton University, Ottawa, ON, Canada K1S 5B6 \\ ${ }^{2}$ School of Electrical Engineering and Computer Science, University of Ottawa, Ottawa, ON, Canada K1N 6N5 \\ ${ }^{3}$ DEI \& Associates Inc., Waterloo, ON, Canada N2L 4E4
}

Correspondence should be addressed to Gandhi Habash; gandhihabash@cmail.carleton.ca

Received 4 September 2014; Accepted 16 November 2014; Published 10 December 2014

Academic Editor: Joydeep Mitra

Copyright (C) 2014 Gandhi Habash et al. This is an open access article distributed under the Creative Commons Attribution License, which permits unrestricted use, distribution, and reproduction in any medium, provided the original work is properly cited.

\begin{abstract}
One of the emerging milestones in building construction is the development of nearly zero energy buildings (NZEBs). This complex concept is defined as buildings that on a yearly average consume as much energy as they generate using renewable energy sources. Realization of NZEBs requires a wide range of technologies, systems, and solutions with varying degrees of complexity and sophistication, depending upon the location and surrounding environmental conditions. This paper will address the role of the above technologies and solutions and discusses the challenges being faced. The objective is to maximize energy efficiency, optimize occupant comfort, and reduce dependency on both the grid and the municipal potable water supply by implementing sustainable strategies in designing a research and sports facility. Creative solutions by the architectural and engineering team capitalize on the design of a unique glazing system; energy efficient technologies; water use reduction techniques; and a combined cooling, heating, and power (CCHP) microgrid (MG) with integrated control aspects and renewable energy sources.
\end{abstract}

\section{Introduction}

The building sector currently accounts for about one-third of the total worldwide energy use and much of this consumption is directly attributed to building design and construction [1] A wide array of measures have been adopted and implemented to actively promote a better energy performance of buildings, including the nearly zero energy building (NZEB) concept, which is a realistic solution for the mitigation of $\mathrm{CO}_{2}$ emissions. The NZED concept is also a viable way of reducing energy use in buildings, in order to alleviate the current worldwide energy challenges of rising prices, climate change, and security of supply [2]. The NZEB implies that the energy demand for electrical power is reduced, and this reduced demand is met on an annual basis from renewable energy supply which can be either integrated into the building design or provided, for example, as part of a community renewable energy supply system. It also implies that the grid is used to supply electrical power when there is no renewable power available and that the building will export power back to the grid when it has excess power generation, in many cases, selling this exported power to the local utility company through a Feed-In Tariff program. The objective of NZEBs is not only to minimize the energy consumption of the building with passive design methods but also to design a building that balances energy requirements with active techniques and renewable technologies.

The increasing number of NZEB demonstration and research projects [3-12] highlights the growing attention given to NZEBs. Goals for the implementation of NZEBs are discussed and proposed at the international level, for example, in the USA within the Energy Independence and Security Act of 2007 (EISA 2007) and at the European level within the recast of the Directive on Energy Performance of Buildings (EPBD) adopted in May 2010. The EISA 2007 authorizes the net zero energy commercial building initiative for all new commercial buildings by 2030. It further specifies a zero energy target for $50 \%$ of US commercial buildings by 2040 and net zero for all US commercial buildings by 2050 [13]. There are also several advanced sustainable 
building design standards such as Ecohomes (BRE, UK), PassivHaus (Germany), and the US Green Building Council's Leadership in Energy and Environmental Design (LEED). These standards provide different ranking criteria to evaluate energy efficiency and/or NZEBs. However, there are no specific strategies or design guidelines provided for achieving NZEB designs. Specific design guidelines and strategies are extremely important for architects or engineers to popularize NZEBs.

Characterizing the energy demand of a building involves the initial identification of specific energy end uses involved including lighting, space heating and cooling, ventilation, water heating, refrigeration, and others such as mechanical and computing systems. Energy optimization (efficiency) of each of the above elements is the first step to address the overall efficiency as the ratio of the energy demand handled by the building to the energy consumed by the building. European energy performance indicators (EPI) [14] and American energy intensities (EI) [15] are valid and synonymous efficiency indicators, since both are ratios of energy use input to energy service output. However, this does not always result in the most optimal overall building performance because several of these functions interact with one another and result in energy wastage due to competing processes.

This work discusses the whole design process of a defined NZEB. First, passive design methods which reduce heating and cooling loads have been investigated. Various energy-efficient techniques including lighting; heating, ventilation, and air conditioning (HVAC); and water performance improvement have been detailed. A combined cooling, heating, and power (CCHP) microgrid (MG) combined with photovoltaic (PV) and wind energy components is integrated in the design of the building to provide additional energy and enable system design optimizations.

The paper is laid out as follows. Section 2 addresses the architectural design aspects of the building. Section 3 discusses the engineering performance aspects including efficient electricity and water usage. Section 4 describes a grid-connected MG for the building with various loads and on-site renewable components. Finally, Section 5 concludes the work presented in the paper.

\section{Architectural Design}

The building chosen to serve as a case study is a sports research facility for the city of Toronto. It is classified by the Ontario Building Code as a Group A, Division 2 occupancy type commercial building under the "education" activity category, which is regarded as one of most energy intensive type after the "office" category.

The main objectives of the design team are to provide interior spaces with as much illumination as possible through natural daylighting, reduce system and plug loads, maximize energy efficiency, optimize occupant comfort, and decrease dependency on both the grid and the municipal potable water supply through integrating leading-edge technologies and NZEB design strategies. The analysis of the case study was performed for one climate scenario: Toronto (Canada), representing a climate with a cold winter. By integrating the above technologies and strategies, the design offsets its dependency on the grid significantly.

2.1. Building Envelope. There are several parameters in a building design that could be controlled to achieve low building energy consumption, including the building orientation and structure (size, layout, partition, etc.), constructions and their materials, whether or not to allow natural ventilation, and natural ventilation control mode [16]. Appropriate siting of the facility has much to do with its energy savings. The footprint is wide and shallow, capitalizing on the unobstructed land mass near Downsview airport. Such a footprint allows natural light to illuminate key areas during daytime hours, thereby reducing the lighting load. Attention has also been paid to the design of the building envelope to maximize thermal performance as a best practice. This is carried out by taking local energy policy, urban planning, and industry standards into account. The building envelope was organized primarily to maintain privacy between different zones. The office and gathering spaces are located at the southern face of the building, capitalizing on daylighting since they will most likely be used during business hours. The research and sports facility components are located along the northern side of the building, since they require more controlled environments and benefit less from natural daylighting and ventilation. The design approach starts by reducing energy demands within the building envelope by minimizing losses and optimizing solar gains. The architectural rendering of the building is shown in Figure 1, while the building major zones are shown in Figure 2.

The target of net zero energy use is a requirement of the living building challenge. Our approach to achieve it is through a "conserve, capture, and create" concept. The heavily articulated building form increases the area of the external envelope with a related increase in heat loss. However, by allowing the building to capture winter solar gain, daylight, and natural ventilation, the final result is a net benefit to the overall energy equation. The south orientation of the glazed entrance wing and some of the classrooms allow lowlevel winter solar gain to be captured, reducing the heating loads on the building. The capture of daylight and natural ventilation reduces lighting and ventilation requirements, further reducing the energy demands of the building.

\subsection{Aspects of Passive Design. When designing NZEB build-} ings, architects control some of the most important aspects of passive design: the geometric expression of massing and program, glazing type, size, assemblies, orientation, and location. These affect nearly every load and energy performance of a building such as heat gain and shading, daylighting, operability, and envelope conduction. Creative solutions by the architectural team capitalized on the large building mass, namely, in the design of a unique glazing system along the south side of the building. The glazing system maximizes daylighting while maintaining occupant comfort. Through the use of three different panels on a pyramid structure, 


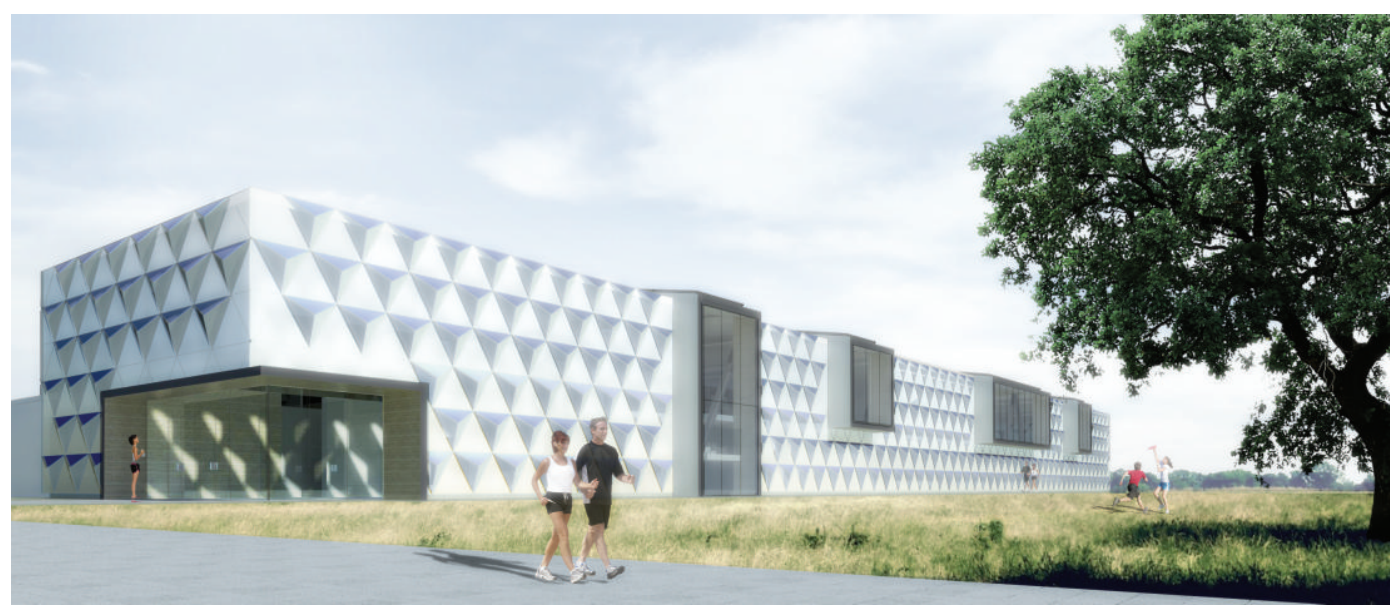

Figure 1: Architectural rendering of the Downsview Plain research and sports building.

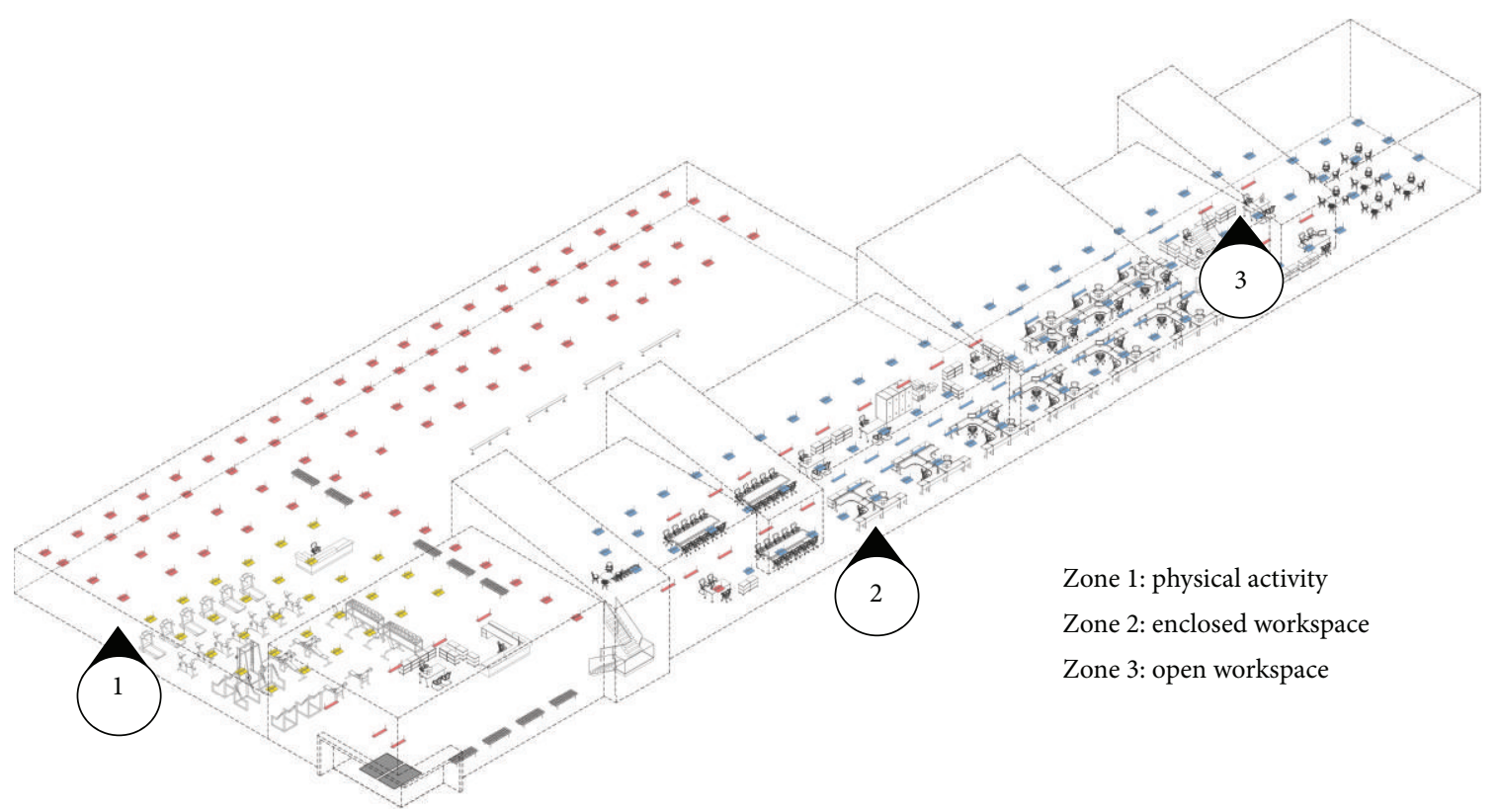

FIGURE 2: The building major zones.

the glazing system minimizes glare and internal heat gains while maximizing light trespass where appropriate. Additionally, the glazing system incorporates PV cells which produce a substantial amount of energy due to their southern orientation.

In NZEB, the roof often becomes the project's densest design challenge in terms of daylight, energy generation, and equipment for photovoltaic system. For the proposed facility, the roof design ingeniously balances the desire for optimally oriented PV panels, skylights, and sun tunnels for daylighting and ventilation.

\section{Engineering Performance Improvement}

There are various energy consuming systems required in a building in order to maintain ideal environmental conditions, including lighting, HVAC loads, environmental loads, and plug loads. The proposed design targets the two largest loads in the building, namely, lighting and HVAC loads, in order to maximize energy savings.

3.1. Metric of Energy Use. Putting metrics on evaluating building energy performance is a necessary step to make any progress on NZEBs. In such evaluation, it is important to consider building use and climate. One useful metric of energy use in buildings is the energy use intensity (EUI). The EUI is typically defined as the ratio of annual site energy use to building floor area. It is calculated by dividing the total energy consumed by the building in one year (measured in $\mathrm{kBtu}$ or GJ) by the total gross floor area of the building. It can be used to refer just to the electricity use in a building in which case it is usually defined in units of $\mathrm{kBTU} / \mathrm{ft}^{2}$ or $\mathrm{kWh} / \mathrm{m}^{2}[17]$. 
3.2. Efficient Lighting System. Lighting is the most pervasive element and is of essential need in modern buildings. It is a significant component of the total energy consumption in a building, often comprising $20-30 \%$ of total building energy consumption. In addition to the advancements in light source technologies, from incandescent and high intensity discharge (HID) to fluorescent and compact fluorescent (CFL) to light emitting diodes (LEDs), controlling the artificial light sources to provide illumination of the right kinds (adequate light levels, colour temperature, and colour rendering), to the right places (offices, recreation areas, etc.) and at the right times, provides significant opportunity for energy savings. The Illuminating Engineering Society (IES) provides a collection of recommended lighting levels for various tasks and occupancy types in the IES Lighting Handbook, but provided that minimum lighting levels and energy targets are met in accordance with the OBC lighting levels are at the final discretion of the building owner and the designers. For this reason, establishing the required lighting levels for each space type then becomes an important design decision, as many of these opportunities for energy savings can only be realized provided that the end users' visual comfort is not negatively affected during standard operating conditions. By completing a photometric study using computer aided modelling software such as AGI32 or Visual 3D, it is possible to design a lighting layout that achieves the desired light levels, while maximizing the energy savings by providing an accurate comparison of fixtures' performances in specific applications and determining optimal fixture placements/aiming within a space. Modelling the lighting also allows the average solar light trespass throughout the days of the year to be taken into account, providing a basis for determining the average contributions from artificial light required to maintain consistent illumination in each space, making it possible to calculate the typical dimming levels for luminaires. Additionally, integrating the lighting control system with the HVAC controls through the Building Automation System (BAS) and with individual plug load controls can provide substantial energy savings and leads the way to the commercial realization of NZEBs.

To estimate power consumption, let $d_{m}$, where $0 \leq d_{m} \leq$ 1 , be the dimming level of the $m$ th LED luminaire. The value $d_{m}=0$ means that the LED is dimmed off completely, whereas $d_{m}=1$ represents that the LED is at its maximum luminance. Dimming of LEDs is typically done using pulse width modulation (PWM) [18], where the dimming level corresponds to the duty cycle of the PWM waveform. Let $d$ be the $M \times 1$ dimming vector of the lighting system given by

$$
d=\left[d_{1}, d_{2}, \ldots, d_{M}\right]^{T}
$$

indicating that the $m$ th LED is at dimming level $d_{m}$. The power consumption of a LED is directly proportional to the dimming level. Denote the average power consumption of the $m$ th LED at dimming level $d_{m}$ by $P_{m}\left(d_{m}\right)$ [19]. Then

$$
P_{m}\left(d_{m}\right)=P_{\mathrm{on}} d_{m}
$$

where $P_{\text {on }}$ is the power consumption of the LED in the onstate.
The occupancy sensing, daylight (photocell) sensing, and communication elements are integral to the lighting control system. In this project, an intelligent lighting system using Zigbee protocol and control capability has been proposed. In addition, luminaires are used to provide the basic function of illumination rendering and for adaptation to sensing information inputs and user preferences. A lighting control system with multitechnology sensors, both standing alone and integral to specific LED luminaires, has been proposed. LEDs are currently the primary illumination source due to their longer lifetimes and better design flexibility. In particular, LED luminaires offer easy and accurate dimming capability. In order to capitalize on the large amount of daylighting afforded by vast glazed areas, an automated control system is employed. The automated lighting control system not only saves energy but also improves indoor environmental quality by reducing unnecessary artificial lighting. The control system uses a network of sensors which automatically control artificial lighting levels depending on the amount of natural sunlight within designated areas.

For the building, the digital addressable lighting interface (DALI) reference design has been adopted. DALI is a concept that stands for an intelligent lighting management system that provides increased energy savings, easier installation and maintenance, and maximum control and retrofit flexibility in an entirely open standard [20]. It is defined in IEC 60929 and has been updated in IEC 62386. One of the main reasons for this update is the inclusion of the LED device type. DALI is an affordable "open systems architecture" that allows any manufacturer's devices to interface with any dimming, control, sensor, or fixture to create a room or area lighting system.

The use of DALI devices with wireless sensor network provides many parameters about the efficient lighting; this is very useful for saving energy and maintenance purposes, as it can detect any single lamp fault allowing a predictive maintenance and group replacement or schedule power consumption rules enabling the integration of the lighting system in the building into smart MG approach, due to monitoring and acting capability.

For simpler projects or for cost savings measures, $0-10 \mathrm{~V}$ dimming control through a Digital Lighting Management (DLM) system is an effective alternative to a DALI control system. Commercially available LED fixtures are increasingly being supplied with $0-10 \mathrm{~V}$ dimming control compatible drivers as a standard feature. $0-10 \mathrm{~V}$ control is another "open systems architecture" and utilizes a DC analogue control signal ranging from $0 \mathrm{~V}$, lowest lumen output, to $10 \mathrm{~V}$, highest lumen output, with the protocol defined by the IEC60929 Annex E standard. This control system was originally developed for dimmable fluorescent fixtures approximately 30 years ago, being adopted into the IEC standards in 1992, but due to the nature of the circuitry within a DC LED driver it is proving to be cost-effective controls solution for LED fixtures as well.

DLM systems consist of local relays packs (or smallscale relay panels), controllers (wall switches/dimmers), and sensors that can either be networked together or act as stand-alone systems. DLM systems providing $0-10 \mathrm{~V}$ control 
TABle 1: Energy-efficient HVAC system and projected energy savings.

\begin{tabular}{ll}
\hline Implementation & Specifications \\
\hline Waste heat recovery system for outside air & $\begin{array}{l}\text { For colder climate applications, outside air ventilation must be preheated } \\
\text { before entering the vents. The waste heat from exhausted air may be used for } \\
\text { such purpose. Accomplished with the integration of a thermal wheel or rotary } \\
\text { heat exchanger with the HVAC system. }\end{array}$ \\
\hline Cooling heat recovery system & $\begin{array}{l}\text { Heat exhausted from a cooling system could be recovered for ventilation and } \\
\text { space heating. Accomplished using heat recovery condensers. }\end{array}$ \\
\hline Air-source heat pump & $\begin{array}{l}\text { Extracts heat from the air and then transfers heat to either the inside or the } \\
\text { outside of your home depending on the season. }\end{array}$ \\
\hline Total projected energy saving & $\mathbf{6 4}$ \\
\hline
\end{tabular}

are readily available, allowing fixtures to be switched and dimmed in control groupings while minimizing wiring by locating the relay packs centrally to the associated fixtures.

The main separation between DLM and DALI systems is that DALI communicates directly with each individual fixture, whereas DLM controls fixtures in groupings. As DLM systems do not communicate directly with each individual fixture there are a number of limitations when compared to a DALI system; namely, there is no mechanism to directly detect faults or maintenance issues, and DLM systems cannot inherently meter each individual fixture's energy usage; however, power monitoring of individual relay packs is achievable as is implementing scheduled power consumption rules. The result of this is overall less flexibility but for most practical applications a very effective solution.

The lighting energy consumption based on DALI/DLM LED industry standard protocol is estimated as $16,418 \mathrm{kWh}$ while the same facility with nonefficient lighting system requires $109,744 \mathrm{kWh}$. Based on the above, the projected energy saving is $84 \%$. Lighting is selected based on energy efficiency, fixture lifespan, and the degree to which the fixture's composition could potentially harm the environment.

A Central Emergency Lighting Inverter (CELI) is proposed to provide the building with an easily maintained and tested emergency lighting system in accordance with the $\mathrm{OBC}$ requirements, providing designated lighting fixtures with battery backed-up AC power produced by a pulse width modulation inverter (creating sinusoidal output at designated voltage from a battery source). The CELI will maintain its charge utilizing locally generated power whenever available. By employing a CELI it is possible to nearly entirely eliminate the need for emergency battery units and remote heads, reducing overall quantity of materials by employing only the fixtures part of the general lighting design to provide emergency lighting as well.

3.3. HVAC Energy Efficiency. Nearly every energy model requires the user to select a HVAC system. The heating and cooling demand is generally driven by the goal of maintaining the occupied space within a comfortable temperature and humidity range. The most widely promoted guidance for thermal comfort comes from the American Society for Heating, Refrigeration and Air Conditioning Engineers (ASHRAE) in the form of the ASHRAE 55 standard for thermal environmental conditions for human occupancy (ANSI/ASHRAE 2010) [17].

Among building energy services, HVAC system is the most energy consuming segment, accounting for about 10$20 \%$ of final energy use in developed countries [21]. The HVAC system generally contains a source of heating and cooling, a distribution system, and a technique for supplying fresh air. These include boilers, furnaces, and electric resistance heaters which are typically used to add heat to buildings while cooling is typically accomplished via air conditioning units, heat pumps, or cooling towers.

While the architect's goal with early design simulation and energy modeling is generally to reduce loads passively, the HVAC system selection and design can have a large impact on resulting energy use. The heating and cooling demand is generally driven by the goal of maintaining the occupied space within a comfortable temperature and humidity range. Ventilation service uses thermal energy for outdoor air treatment and electrical energy for filtration and distribution to conditioned spaces.

The number of occupants needs to be defined so as to determine the hot water needs as well as the thermal internal gains. The cooling oversize recommended for the building is estimated as 581,625 Btuh [22]. This includes base cooling and additional cooling required for an assumed occupancy of 75 people and 4 kitchens, as proposed in the case study. Accordingly, 5 rooftop units are recommended to share the load with supplemental electric heating. To minimize the building's heating and cooling energy consumption, two systems were integrated into the HVAC design. First, an airsource heat pump is used to extract heat from the outdoor air, resulting in $15 \%$ of additional energy saving. Second, waste heat and cooling recovery systems are used to preheat fresh outdoor air. The largest percentage of energy savings comes from the specification of energy-efficient HVAC equipment. Implementing these systems results in an overall savings of $64 \%$ when compared to traditionally designed systems as shown in Table 1.

3.4. Water Performance Improvement. Externally, considerations for irrigation have been completely eliminated through lower-technology, low-cost, and high-impact strategies such as xeriscaping. Native plant species and natural habitat are maintained and promoted throughout the site, thus saving 


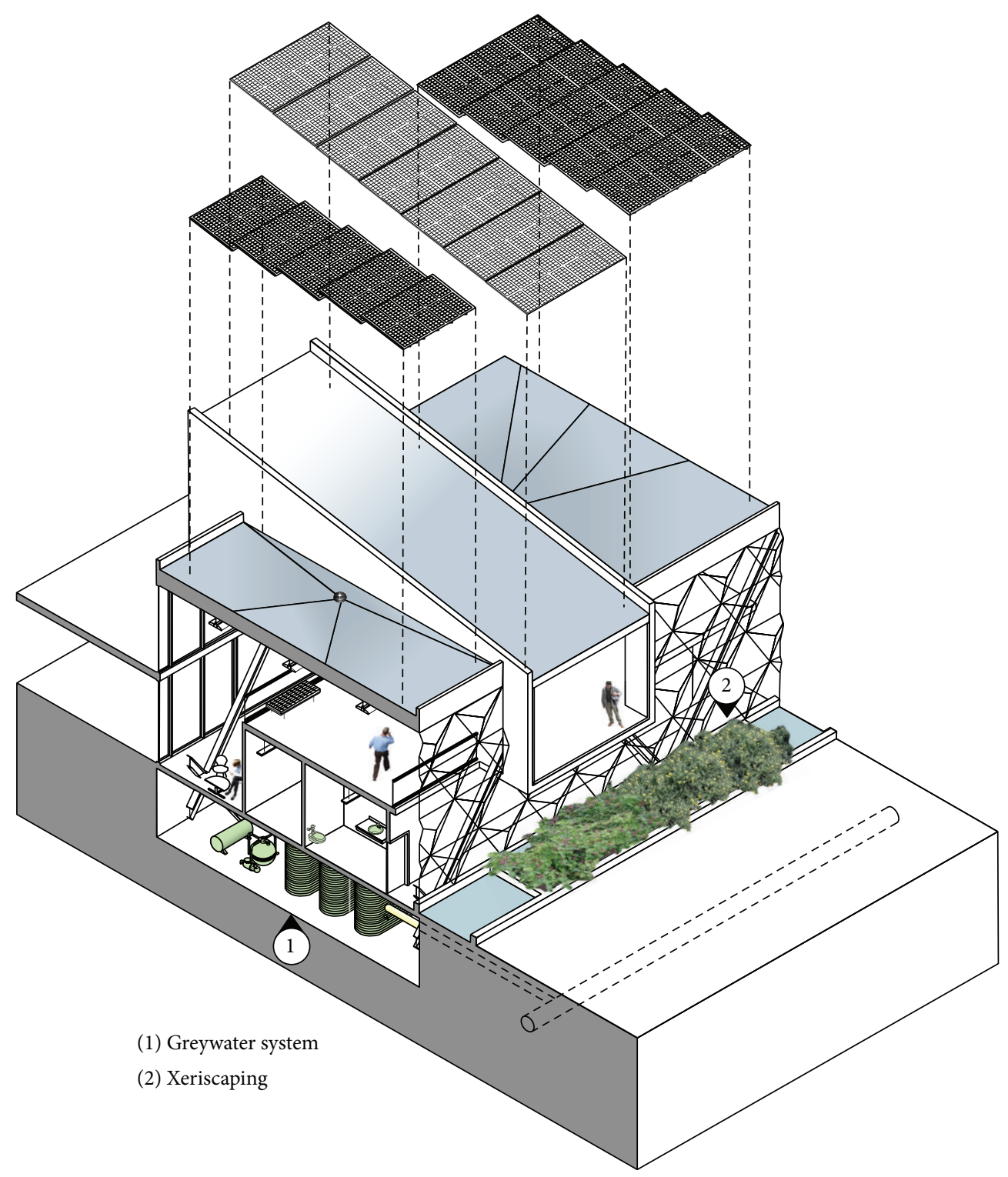

FIgURE 3: Water use reduction proposal for the building.

tens of thousands of gallons of water annually when compared to traditional landscaping techniques.

Internally, the full-time equivalent (FTE) for the building was calculated at 38 FTE including closed offices, workstations, and hoteling stations based on 250 days worked per year. Using default EPAct figures for flush and flow fixtures, the baseline value for daily water use was calculated as 55,425 gals/year. While substituting the EPAct flush and flow rates with flush and flow rates for water-efficient fixtures (waterless urinals, dual-flush toilets, and low-flow lavatories) the baseline water use was reduced to 29,687 gals/year. This reduces the need for municipally provided potable water by $54 \%$ annually.

As a result of the above considerations, the proposal is more healthful, energy-efficient, and sustainable alternative to a traditionally built complex. Water saving strategies such as the incorporation of water-efficient fixtures typically have little added cost. Figure 3 shows the water use reduction proposal for the building.

\section{CCHP Microgrid}

MG is the cornerstone and indispensable infrastructure of smart grid [23]. It is defined as a cluster of loads and energy generation sources operating as a single controllable system that provides both power and heat to its local area [24, 25]. Development of the CCHP MG by using various loads and renewable energy sources has drawn considerable research attention recently. Compared with conventional CCHP systems, the CCHP MG has novel and greater functionality, because the CCHP MG not only satisfies the cooling, heating, and power demands of buildings but also interacts with 


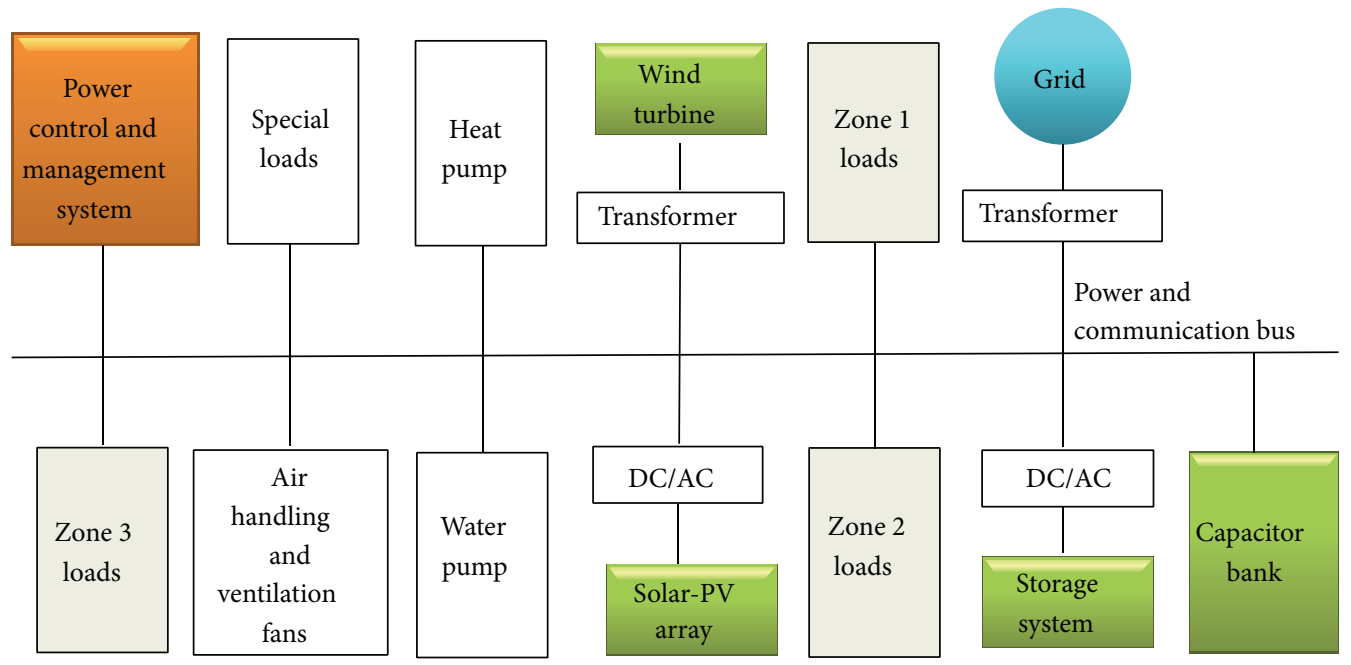

FIGURE 4: Schematic of the proposed building CCHP MG.

the main grid to provide reserve, peak-saving, and demand response services and provides improved capability for integration of renewable energy sources [26].

MGs, if designed properly, are capable of operating in either grid-connected mode or islanded mode [27]. MG technology provides an opportunity and a desirable infrastructure for improving the efficiency of energy consumption in buildings [28]. Recent research work shows that $20 \%-$ $30 \%$ of building energy consumption can be saved through optimized operation and management without changing the structure and hardware configuration of the building energy supply system [29].

The majority of electrical loads within a building can be grouped into lighting subsystems, computing equipment, individual plug-loads, and HVAC related equipment. All these subsystems must be optimized to improve the energy efficiency of the building. A schematic of the MG model proposed for the building is shown in Figure 4. Through optimal operation control of the MG, this system will enable the building to maximize the gain of the renewable sources, to improve energy efficiency, to decrease the energy bill, and to reduce greenhouse gas emission.

4.1. Annual Energy Demand. As opposed to residential buildings, commercial buildings have energy consumption profiles that are driven primarily by the defined work week. That means having very low occupancies on weekends, holidays, early morning hours, and weekday evenings. Characterizing the energy demand of a building involves firstly identifying the specific energy end uses involved. Each major end use that forms the energy demand can be influenced by a number of design variables, and each building design variable has a wide range of likely values or alternatives. The load profile of the facility is proposed to closely match that of similar typical buildings for which a measurement campaign was undertaken. Figure 5 illustrates the projected power demand of the building. The annual energy demand for the building was estimated by making seasonal adjustments to the weekly

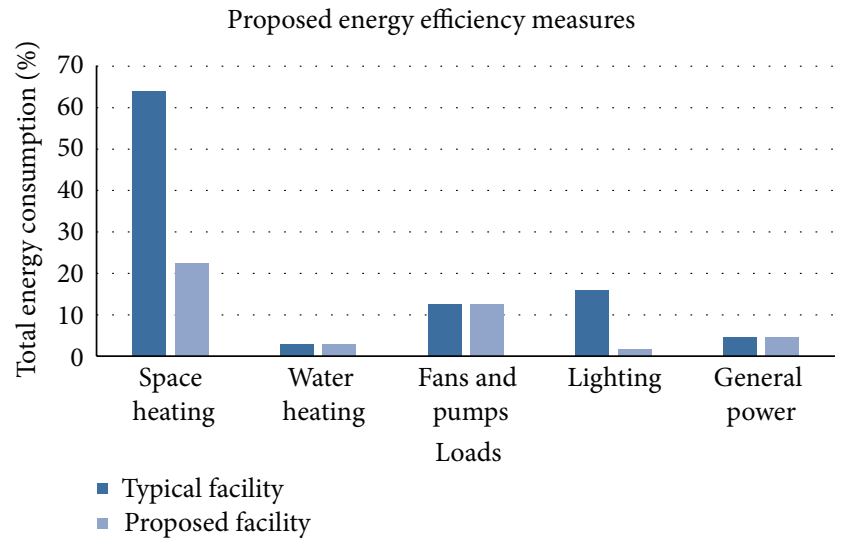

FIGURE 5: Load distribution of the building: typical versus proposed.

demand and typical day profiles and summating it over a year. The total power consumption in various components of the building (sport performance laboratory, strength and conditioning facility, ancillary rooms, reception, server room, showers/locker rooms/washrooms, offices, boardrooms, hoteling stations, staff and demonstration kitchens, athlete career lounge, hallways, track, and field floodlights) has been estimated based on size, lumens/luminary, type of fixtures, utilization factor, maintenance factor, and lighting on 18 hours/day. Based on the above estimation, an expected total approximated energy demand of $336.79 \mathrm{MWh}$ per year was established.

4.2. On-Site Renewable Energy. A number of factors are considered to estimate the amount of solar energy available on a building. First is the area of roof that is available to install solar-PV array. Due to the placement of mechanical systems on the roof, the available area is often less than the actual roof area. One way to increase it is by installing solar-PV array on the wall. Besides the amount of space available, other 
TABLE 2: Technical details of the three locations of solar-PV array.

\begin{tabular}{|c|c|c|c|c|c|c|c|}
\hline $\begin{array}{l}\text { Solar-PV } \\
\text { location }\end{array}$ & Racking type & Tilt (degree) & $\begin{array}{l}\text { Number of } \\
\text { solar-PV } \\
\text { modules }\end{array}$ & $\begin{array}{l}\text { Module area } \\
\qquad\left(\mathrm{m}^{2}\right)\end{array}$ & $\begin{array}{c}\text { Nominal power } \\
(\mathrm{kW})\end{array}$ & $\begin{array}{l}\text { Performance } \\
\text { ratio }(\%)\end{array}$ & $\begin{array}{c}\text { Yearly } \\
\text { production } \\
(\mathrm{MWh} / \text { year })^{2}\end{array}$ \\
\hline Solar wall & $\begin{array}{l}\text { Building } \\
\text { integrated }\end{array}$ & 36.86 & 85 & 137 & 21.68 & 79.4 & 25.45 \\
\hline Sloped roof & $\begin{array}{l}\text { Extruded } \\
\text { aluminum }\end{array}$ & 10 & 234 & 376 & 59.67 & 81.5 & 68.30 \\
\hline Flat roof & $\begin{array}{l}\text { Ballasted KB } \\
\text { EKONORACK }\end{array}$ & 10 & 740 & 1190 & 188.70 & 77.6 & 206.00 \\
\hline & Total & & 1059 & 1703 & 270.05 & 78.6 & 299.75 \\
\hline
\end{tabular}

${ }^{1}$ Actual number of modules is based on the area.

${ }^{2}$ Yearly production values are based on simulation.

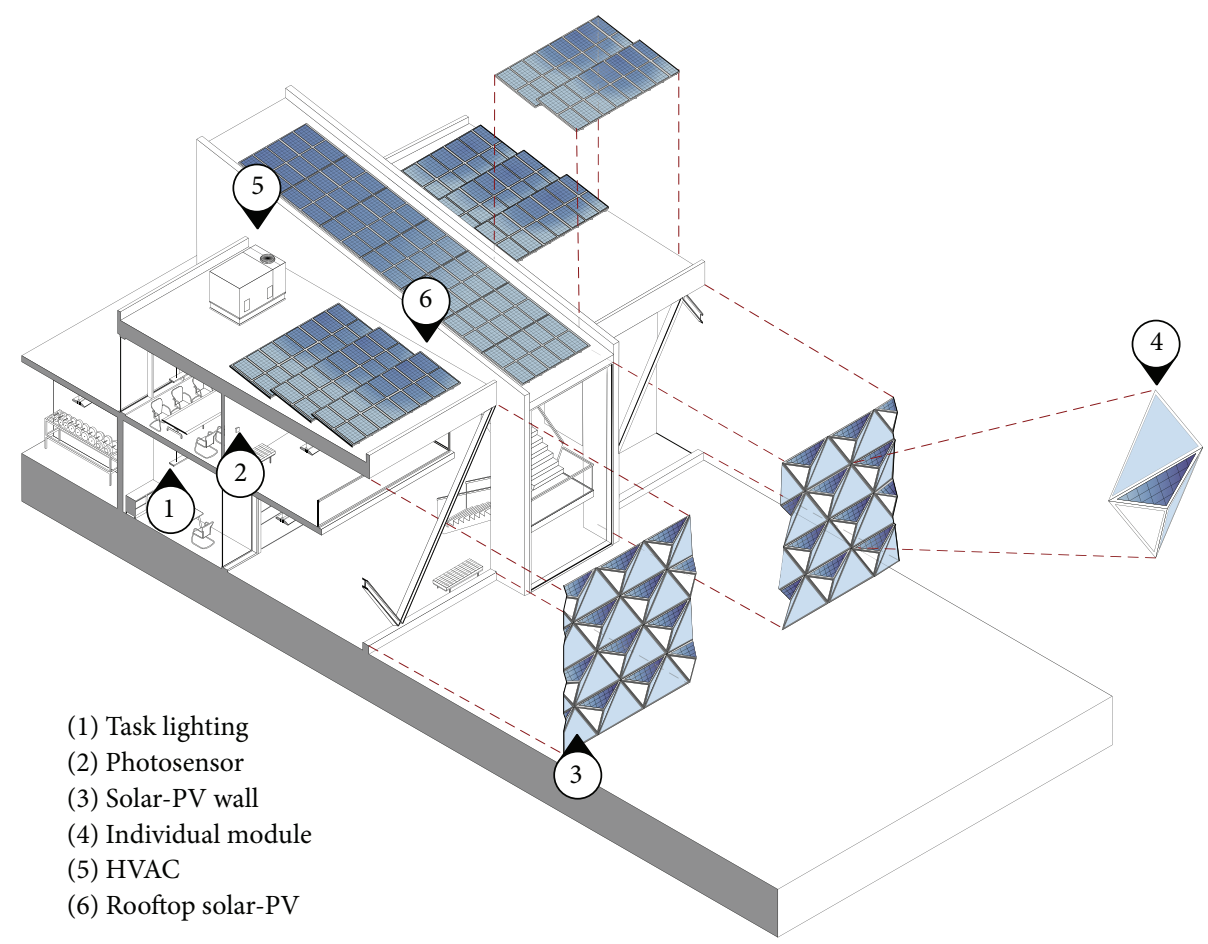

FIGURE 6: Solar-PV array in the building.

important factors include the amount of energy production available from the solar panels themselves. This depends on the efficiency of the panels and the amount of solar radiation exposure. For this project, solar-PV array is proposed on three parts of the building including the triangular sections on the wall and sloped and flat roofs as shown in Figure 6. Based on photovoltaic software calculations, the total estimated capacity for the three solar-PV arrays is $270 \mathrm{~kW}$ as shown in Table 2. This includes margins for factors such as panel availability, load estimation, equipment efficiency, and solar-PV array and inverter losses. The energy produced by the solar-PV array allows the building to achieve a total value of $89 \%$ energy offset.

The generating resources in the proposed MG include a solar-PV array and a small-scale wind generator. The wind turbine associated with the MG is treated as an experimental generation unit and thus was not included in the energy calculations. A small-scale contrarotating induction generatorbased wind turbine is proposed with a nominal rating of $75 \mathrm{~kW}$ [30]. Its presence provides some additional margin for meeting the energy requirements and also assists in eliminating required energy storage. It is anticipated that the proposed wind turbine will provide approximately $340 \mathrm{MWh}$ per year at a wind speed of $7.5 \mathrm{~m} / \mathrm{s}$ [31]. The proposed MG is expected to operate in either grid-connected mode or islanded mode in order to maximize the energy harvesting from the solar-PV array and ensure economically feasible operation.

4.3. Energy Storage System. Energy storage is designed not only to conserve energy but also to allow demand shifting where energy can be stored during lower demand times and 
used during peak demand periods. Several strategies can be considered when sizing on-site energy storage including peak demand reduction, energy harvesting to reduce export, mitigating intermittent grid outages, and standalone operation. Each strategy has differing demands on energy storage sizing and economic constraints. For the proposed facility, sizing energy storage for prolonged intentional islanding is not required, and in order to minimize the energy storage requirement (due to financial constraints), intentional islanded operation is limited to a single day demonstration.

There are several forms of energy storage available that can be used in MGs. These include batteries, supercapacitors, and flywheels. Conventional lithium-ion batteries are the selected energy storage technology for the building due to their high density (90-150 Wh/kg), high efficiency ( $80-85 \%$ DC to DC), and long cycle life (3000 cycles at $80 \%$ DOD). The main disadvantages are the high cost (about $\$ 600 / \mathrm{kWh}$ ), complex battery management circuitry, and safety matters related to thermal management [25]. For preliminary design, $150 \mathrm{kWh}$ is selected as the capacity, with intentional transition to islanded mode dependent on state of charge (SOC), available generation (weather forecast), and load shedding. Extending islanded mode operation in the future may be made possible by increasing energy storage or localized generation.

4.4. Capacitor Bank. Shunt capacitor banks are used to improve the quality of the electrical supply and the efficient operation of the power system. To meet utility connection requirements, the reactive power demand of a building is compensated using dynamically switched banks to provide capacitive reactive compensation/power factor correction (PFC). Eight segmented PFC stages are proposed for the building. The peak reactive power demand of the sample building is able to be delivered by the first six segments of PFC capacitors, for example, $60 \mathrm{kVAR}$. An additional $20 \mathrm{kVAR}$ segment is provided to deliver margin for motor start requirements of additional equipment for the building.

4.5. Surge Protective Devices (SPDs). The sophistication of the electrical equipment to be installed on this and other similar projects is achieved mostly through the introduction of microprocessors/microcontrollers as control units for the variety of electrical devices such as LED drivers, lighting control systems, and variable-frequency drives. By implementing these microelectronic devices, this equipment has become as sensitive to transient voltages and voltage surges as any computer load. In order to protect the significant investment this equipment represents as much as possible, surge protective devices (SPDs) in a tiered approach have been proposed. The tiered approach refers to the implementation of SPDs on each level of distribution, that is, the facilitywide protection on the service entrance switchboard and the branch level protection on the individual panel boards. This tiered approach allows for externally and internally generated surges and transients to be reduced to harmless levels by knocking the impulse waveforms down in stages based on amplitudes, greatly reducing their impact on the desired waveforms and leaving no single point of exposure upon failure of an individual SPD.

4.6. DC Bus. In the future, it is worthwhile to consider a DC bus to supply the required power to the appliances where MGs employing renewable energy sources and LED lighting with a large fraction of appliances are internally DC powered. This would eliminate the need for DC to AC and back from $\mathrm{AC}$ to $\mathrm{DC}$ conversions, thereby saving energy on the whole. The literature shows that a large segment of the building's electric load could be fed directly with DC power including devices based on microprocessors, computer system power supply, switched-mode power supply, electronic ballasts for the fluorescent lighting, variable-frequency drives for the speed variation of the motors which equip the systems of HVAC, and lighting based on LEDs [32-34].

4.7. Control and Management. The MG control and management system synchronizes multiple energy sources with the grid and with building loads. The MG is based on key components including a local monitoring and control system, communication network, and central controller integrated with an intelligent energy management system (IEMS). The controller consists of algorithms for real-time monitoring and control of the network. Sources and loads need to communicate quickly with each other. This is an opportunity for short messaging protocols such as extensible messaging and presence protocol (XMPP) which is widely used by Twitter, Google Talk, Facebook, and other large scale applications. XMPP may use a connection through a cloud server to link an external energy source management network with an internal building load management network. The integrated IEMS regulates energy consumption and storage according to the availability of its resources.

Electricity and cold and heating demands fluctuate both daily and seasonally. Therefore, taking operational strategies into account during the design stage should prove beneficial for the candidate CCHP system. Consequently, a robust technique for sizing and identification of the optimum operational strategies for such systems is needed [25].

Requirements for control systems to achieve efficient performance and regulation of various facilities can be organized in several categories including zone controls (thermal zoning, zone isolation, temperature, and humidity controls); air handling unit controls (variable air volume and temperature); water loop controls (pump isolation and load management); primary equipment controls (cooling towers and heat pumps); and automatic meter reading and data collection facilities. Therefore, intelligent control of the above elements offers additional energy savings otherwise not possible with independent operation.

\section{Concluding Remarks}

The rising problems of energy shortages and environmental concerns have boosted the development of the NZEBs and CCHP MGs. As a consequence of the expected widespread development of NZEBs and CCHP MGs, there is an urgent 
need to improve the available interdisciplinary skills for design and operation of the systems to realize energy savings, environmental protection, and economical operation. In this context, this paper presented a methodology with the goal of assisting the choice of economically efficient NZEB and CCHP MG solutions, right from the early design stage. This can be used for any residential or commercial building in any part of the world considering the local climate, the endogenous energy resources, and the local economic conditions that lead to a nearly zero annual energy balance. Achieving nearly zero is not only a matter of design; it requires careful attention to operations and maintenance and to occupancy patterns and loads. While such buildings are possible with existing technologies, this research uncovered the challenges associated with achieving nearly zero energy in this building with today's onsite renewable energy and MG technologies. However, the encouragement of nearly zero at the building scale sets the stage for future technology solutions and the removal of barriers to energy and water systems to move buildings to energy and water independence.

The objective of this work is to maximize energy efficiency, optimize occupant comfort, and reduce dependency on both the grid and the municipal potable water supply by implementing sustainable strategies in designing a research and sports building. Creative solutions by the architectural and engineering team capitalize on the design of a unique glazing system; energy-efficient technologies; water use reduction technique; and a CCHP MG with integrated control aspects and renewable energy component. The CCHP MG provides an effective solution to energy-related problems, including increasing energy demand, higher energy costs, energy supply security, and environmental concerns. The key challenge to improve building energy efficiency in operation is to coordinate and optimize the operation of various energy sources and loads. As a result of the above considerations, the proposed building is a more healthful, energy-efficient, and sustainable alternative to a traditionally built complex.

It must nevertheless be noted that the design tool presented in this paper intends to provide guidance in the early design stage more than to replace a detailed dynamic assessment of the solution that ends up being elected by the architecture and engineering design teams.

\section{Conflict of Interests}

The authors declare that there is no conflict of interests regarding the publication of this paper.

\section{Acknowledgments}

The authors are grateful to Royal Bank of Canada (RBC) Foundation and $\mathrm{B}+\mathrm{H}$ Architects for the opportunity to participate in the annual Evolve Sustainable Design Competition and for their financial support.

\section{References}

[1] IEA (International Energy Agency), "Energy Balances of OECD Countries-2010 edition," Paris, France, 2010.

[2] A. J. Marszal, P. Heiselberg, J. S. Bourrelle et al., "Zero Energy Building - a review of definitions and calculation methodologies," Energy and Buildings, vol. 43, no. 4, pp. 971-979, 2011.

[3] P. Torcellini, S. Pless, and M. Deru, "Zero energy buildings: a critical look at the definition," in Proceedings of the ACEEE Summer Study on Energy Efficiency in Buildings, Pacific Grove, Calif, USA, August 2006.

[4] M. Noguchi, A. Athienitis, V. Delisle, J. Ayoub, and B. Berneche, "Net zero energy homes of the future: a case study of the EcoTerraTM house in Canada," in Renewable Energy Congress, Glasgow, Scotland, July 2008.

[5] "The Active House project," http://www.activehouse.info.

[6] M. Heinze and K. Voss, "Goal: Zero energy buildingexemplary experience based on the solar estate solarsiedlung freiburg am schlierberg, Germany," Journal of Green Building, vol. 4, no. 4, pp. 93-100, 2009.

[7] E. Musall, T. Weiss, A. Lenoir, K. Voss, F. Garde, and M. Donn, "Net zero energy solar buildings: an overview and analysis on worldwide building projects," in Proceedings of the EuroSun Conference, Graz, Austria, 2010.

[8] E. Doub, Solar Harvest: City of Boulder's First Zero Energy Home, Ecofutures Building, Boulder, Colo, USA, 2009.

[9] R. Hawkes, Crossway Eco-House, Hawkes Architecture, Kent, UK, 2009.

[10] K. B. Wittchen, J. S. Ostergaard, S. Kamper, and L. Kvist, BOLIG+ an Energy Neutral Multifamily Building, EuroSun, Graz, Austria, 2010.

[11] M. Kapsalaki and V. Leal, "Recent progress on net zero energy buildings," Advances in Building Energy Research, vol. 5, no. 1, pp. 129-162, 2011.

[12] A. Ferrante and M. T. Cascella, "Zero energy balance and zero on-site $\mathrm{CO}_{2}$ emission housing development in the Mediterranean climate," Energy and Buildings, vol. 43, no. 8, pp. 20022010, 2011.

[13] D. Crawley, S. Pless, and P. Torcellini, "Getting to net zero," ASHRAE Journal, vol. 51, no. 9, pp. 18-25, 2009.

[14] EN 15217, "Energy performance of buildings-methods for expressing energy performance and for energy certification of buildings," in European Committee for Standardization, 2007.

[15] Energy Information Administration (EIA), Measuring Energy Efficiency in the United States Economy: A Beginning, Department of Energy, 1995.

[16] H. Liu, Q. Zhao, N. Huang, and X. Zhao, "A simulationbased tool for energy efficient building design for a class of manufacturing plants," IEEE Transactions on Automation Science and Engineering, vol. 10, no. 1, pp. 117-123, 2013.

[17] D. J. Sailor, "Energy buildings and urban environment," in Vulnerability of Energy to Climate, vol. 3, pp. 167-182, 2013.

[18] Y. Gu, N. Narendran, T. Dong, and H. Wu, "Spectral and luminous efficacy change of high-power LEDs under different dimming methods," in Sixth International Conference on Solid State Lighting, vol. 6337 of Proceedings of SPIE, pp. 63370J-163370J-7, 2006.

[19] A. Pandharipande and D. Caicedo, "Adaptive illumination rendering in LED lighting systems," IEEE Transactions on Systems, Man, and Cybernetics, vol. 43, no. 5, pp. 1052-1062, 2013. 
[20] M. Moeck, "Developments in digital addressable lighting control," Journal of Light and Visual Environment, vol. 28, no. 2, pp. 104-106, 2004.

[21] L. Pérez-Lombard, J. Ortiz, and C. Pout, "A review on buildings energy consumption information," Energy and Buildings, vol. 40, no. 3, pp. 394-398, 2008.

[22] Natural Resources Canada, Air-Source Heat Pumps.

[23] X. Tan, Q. Li, and H. Wang, "Advances and trends of energy storage technology in Microgrid," International Journal of Electrical Power and Energy Systems, vol. 44, no. 1, pp. 179-191, 2013.

[24] W. Y. Habash, V. Groza, T. McNeill, and I. Roberts, "Lightning risk analysis of a power microgrid," British Journal of Advanced Science and Technology, vol. 3, no. 1, pp. 107-122, 2013.

[25] S. X. Chen, H. B. Gooi, and M. Q. Wang, "Sizing of energy storage for microgrids," IEEE Transactions on Smart Grid, vol. 3, no. 1, pp. 142-151, 2012.

[26] W. Gu, Z. Wu, R. Bo, W. Liu, G. Zhou, and W. Chen, "Modeling, planning and optimal energy management of combined cooling, heating and power microgrid: a review," International Journal of Electrical Power and Energy Systems, vol. 54, pp. 2637, 2014.

[27] L. G. Meegahapola, D. Robinson, A. P. Agalgaonkar, S. Perera, and P. Ciufo, "Microgrids of commercial buildings: strategies to manage mode transfer from grid connected to islanded mode," IEEE Transactions on Sustainable Energy, vol. 5, no. 4, pp. 13371347, 2014.

[28] R. O’Neill, “Smart grids: sound transmission investments," IEEE Power and Energy Magazine, vol. 5, no. 5, pp. 101-104, 2007.

[29] X. Guan, Z. Xu, and Q.-S. Jia, "Energy-efficient buildings facilitated by microgrid," IEEE Transactions on Smart Grid, vol. 1, no. 3, pp. 243-252, 2010.

[30] R. W. Y. Habash and P. Guillemette, "Harnessing the winds: trends and advances," IEEE Potentials, vol. 31, no. 1, pp. 16-21, 2012.

[31] http://www.greenengineers.ca/REMLab/ZECWP\%20-\%20customer\%20Package\%20v5.3.pdf.

[32] K. Engelen, E. L. Shun, P. Vermeyen et al., "The feasibility of small-scale residential DC distribution systems," in Proceedings of the 32nd Annual Conference on IEEE Industrial Electronics (IECON '06), pp. 2618-2623, November 2006.

[33] D. Salomonsson and A. Sannino, "Low-voltage DC distribution system for commercial power systems with sensitive electronic loads," IEEE Transactions on Power Delivery, vol. 22, no. 3, pp. 1620-1627, 2007.

[34] M. Sechilariu, B. Wang, and F. Locment, "Building-integrated microgrid : advanced local energy management for forthcoming smart power grid communication," Energy and Buildings, vol. 59, pp. 236-243, 2013. 


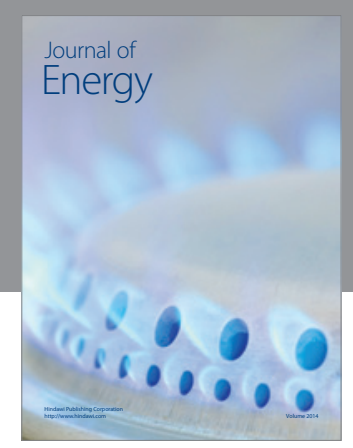

Journal of

Industrial Engineering
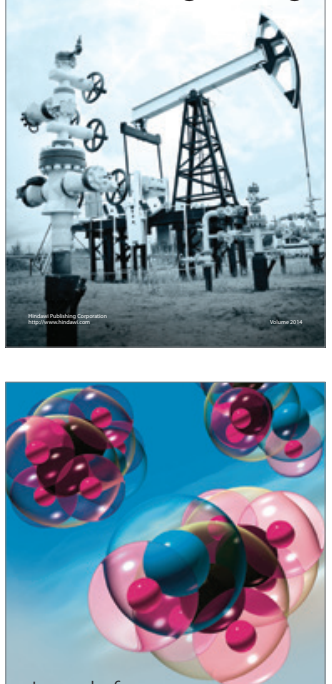

Fuels
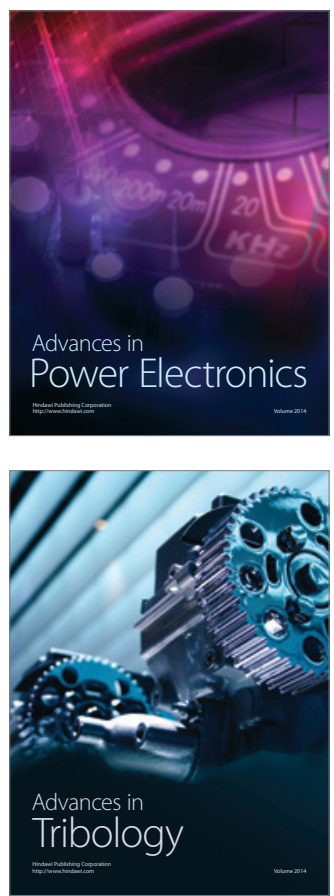

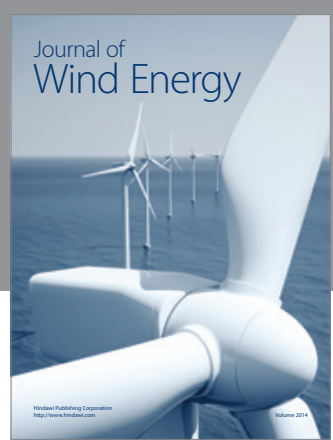

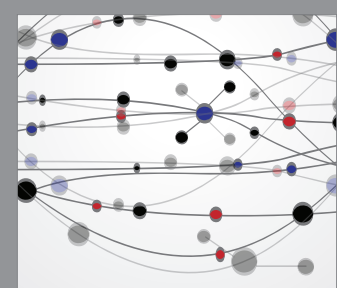

The Scientific World Journal

Submit your manuscripts at http://www.hindawi.com

Journal of

Structures
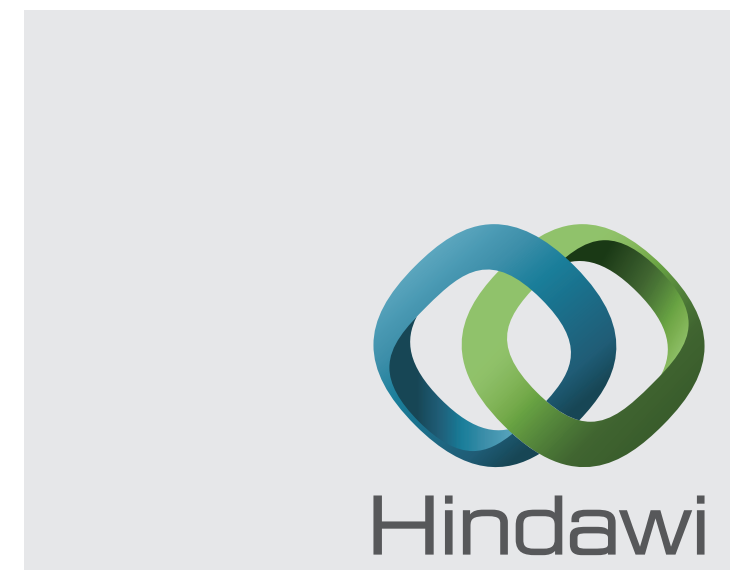

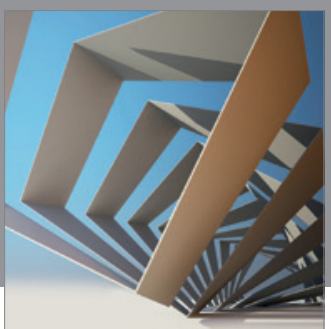

Rotating

Machinery
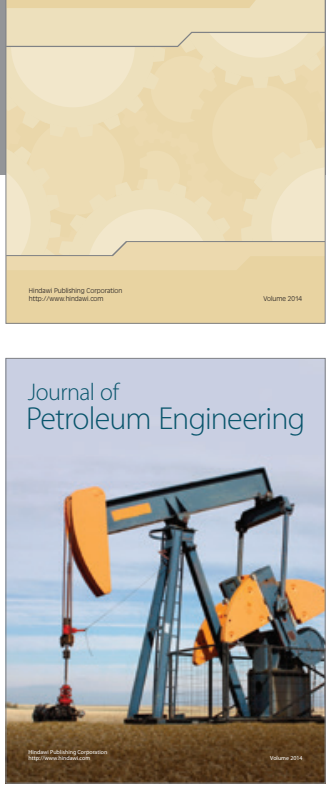

Journal of

Solar Energy
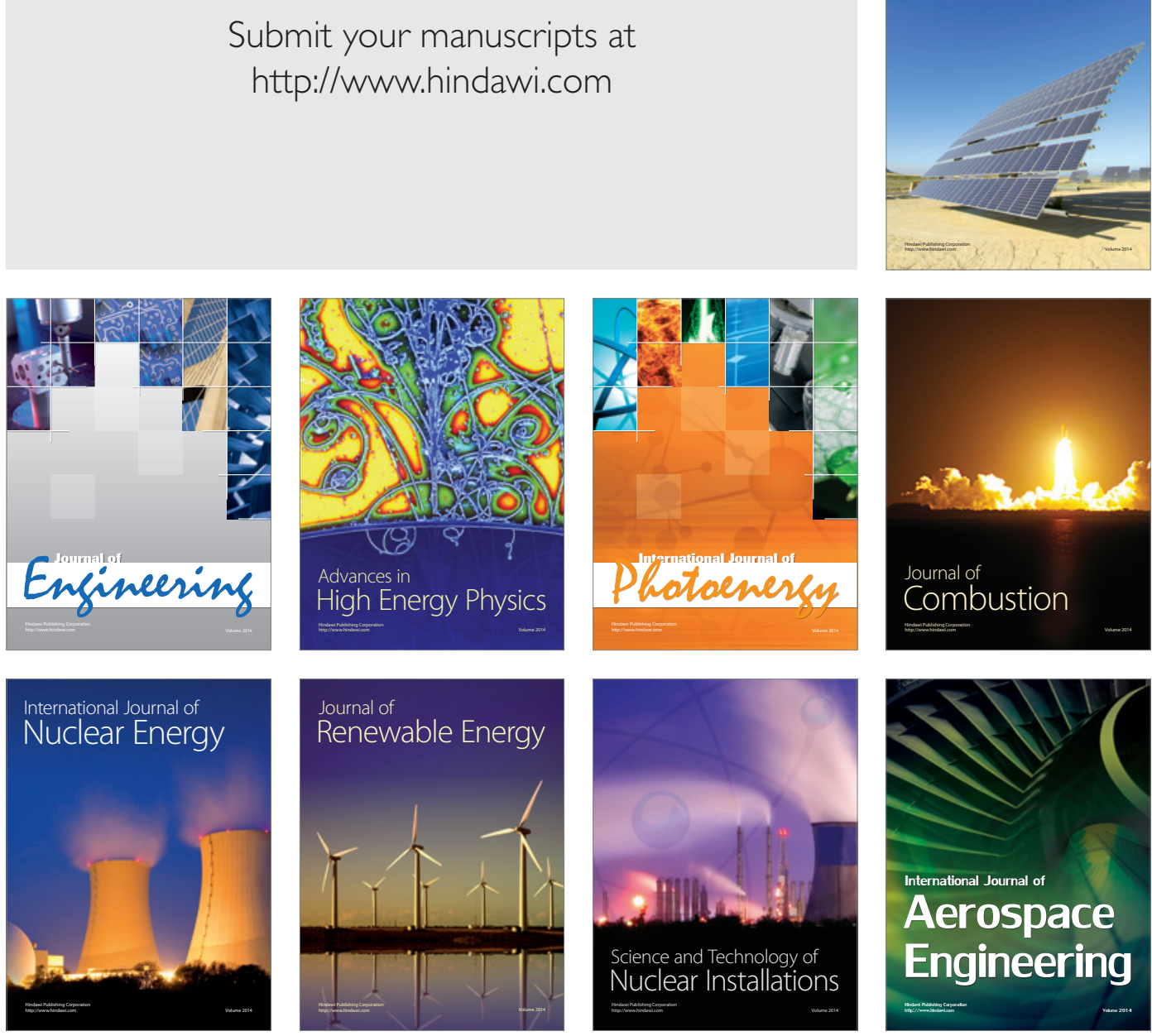